\title{
Cardiovascular and Renal Links along the Cardiorenal Continuum
}

\author{
José A. García-Donaire and Luis M. Ruilope \\ Hypertension Unit, Hospital 12 de Octubre, Avenida Córdoba s/n, 28041 Madrid, Spain \\ Correspondence should be addressed to José A. García-Donaire, docdonaire@terra.es
}

Received 17 August 2010; Accepted 7 February 2011

Academic Editor: C. Ronco

Copyright ( 2011 J. A. García-Donaire and L. M. Ruilope. This is an open access article distributed under the Creative Commons Attribution License, which permits unrestricted use, distribution, and reproduction in any medium, provided the original work is properly cited.

\begin{abstract}
The cardiorenal syndrome includes the widely known relationship between kidney function and cardiovascular disease. A large number of patients have various degrees of heart and kidney dysfunction worldwide, both in developed and developing countries. Disorders affecting one of them mostly involve the other. Such interactions represent the pathogenesis for a clinical condition called cardiorenal syndrome. Renal and cardiovascular disease shares similar etiologic risk factors. The majority of vascular events are caused by accelerated atherosclerosis. Moreover, cardiovascular events rarely occur in patients without underlying disease; rather, they typically take place as the final stage of a pathophysiological process that results in progressive vascular damage, including vital organ damage, specifically the kidney and the heart if these factors are uncontrolled. Chronic kidney disease is a novel risk factor included at this stage that accelerates both vascular and cardiac damage.
\end{abstract}

\section{Introduction}

The interaction between renal and cardiac function is very important for regulatory functions and hemodynamic control. The kidney plays the central role for body fluid volume homeostasis, electrolyte balance, and blood pressure regulation [1]. The relationship between heart and kidney occurs at multiple levels, including the rennin-angiotensinaldosterone system (RAAS), the sympathetic nervous system (SNS), natriuretic peptides, endothelin, and antidiuretic hormones [2]. Therefore, understanding these two important systems is crucial to improve the management of patients with cardiorenal disease. An aging population and increasing incidence of hypertension, type 2 diabetes mellitus, obesity, and other cardiovascular $(\mathrm{CV})$ risk factors are associated with an increasing incidence of cardiorenal disorders. Hence, it is not surprising that the prevalence of heart failure and chronic kidney disease (CKD) continues to increase. Furthermore, it has been shown that even mild-to-moderate deterioration of kidney function correlates with higher morbidity and mortality in patients with heart failure and acute coronary syndrome [3]. The strong relationship between CKD and accelerated CV disease morbidity and mortality has been shown in several epidemiologic data and clinical studies [4]. Moreover, whereas death rates from coronary artery disease have fallen by $35 \%$ in the last decade as a consequence of control of $\mathrm{CV}$ risk factors and optimal therapeutic management, patients with CKD have not accomplished that trend during that period. A significant number of patients with $\mathrm{CKD}$ die of $\mathrm{CV}$ complications before they progress to end-stage renal disease (ESRD), and renal dysfunction in patients with primary cardiac disease portends a significantly enhanced risk of morbidity and mortality from CVD [5]. Thus, with the aging of the population and control of CV risk factors, especially arterial hypertension, understanding the mechanisms of renal dysfunction as a pathogenic factor for cardiovascular (CV) disease is imperative.

\section{Pathophysiological Mechanisms Underlying the Cardiorenal Disease}

CVDs are a leading cause of death and serious morbidity or disabilities worldwide, and CV events rarely occur in patients without underlying disease; rather, they typically take place as the final stage of a pathophysiological process that results 


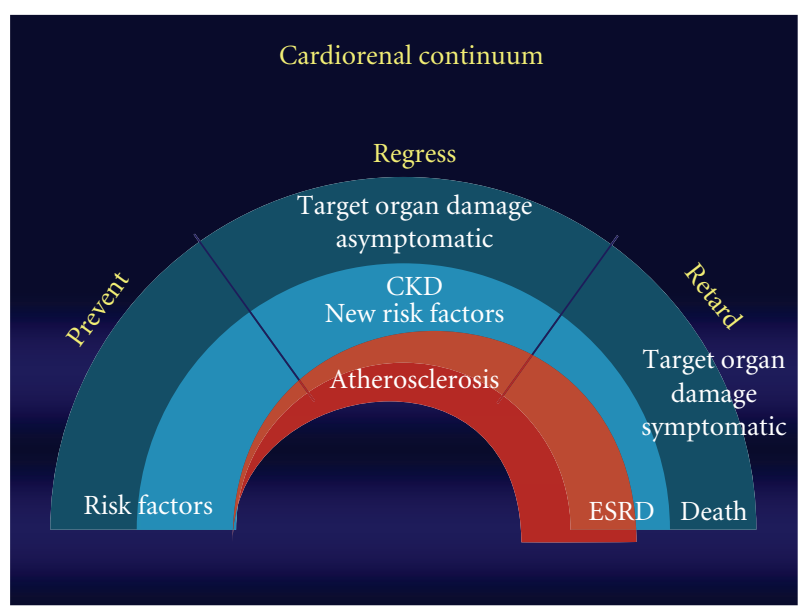

FIGURE 1: Graphic representation of the cardiorenal continuum.

in progressive vascular damage. This stage is called the cardiorenal continuum [6]. Figure 1 displays an overview of the cardiorenal continuum, illustrating a simplified version of the sequential occurrence of the atherosclerotic process from the first stage, in which CVD risk factors are detected and can be prevented if the conditions are appropriately controlled by implementing the optimal therapeutic approaches. A consensus conference has recently presented a classification of cardiorenal disease, including a division of five subtypes of cardiorenal syndromes, according to their pathophysiological mechanisms [7].

Renal and CV diseases share the same etiopathogenic risk factors, including hypertension, dyslipidemia, glucose metabolism disturbances, cigarette smoking, obesity, and physical inactivity. If these factors are controlled, then atherosclerotic process evolution and further target-organ damage (TOD) or CV events can be prevented. Therefore, prevention can be carried out not just at the first stage but along the whole continuum. As the cardiorenal process advances, atherosclerotic vascular damage progresses, and subclinical organ damage can be detected. This is an intermediate stage in the continuum of vascular disease and a determinant of overall CVD risk. CKD is included at this stage, and a number of conditions associated with renal-function decline, such as anemia, secondary hyperparathyroidism, or accumulation of atherogenic substances, become new CVD risk factors and accelerate vascular disease. Therapeutic approaches at this point can regress $\mathrm{CV}$ damage, as shown in the Losartan Intervention for Endpoint Reduction in Hypertension (LIFE) study, in which reduced urinary albumin/creatinine ratio (UACR) and regression of left ventricular hypertrophy (LVH) were associated with lower incidence of CV events. Therefore, strict objectives regarding CVD risk factors must be set up. A large body of evidence is now available concerning the crucial role of TOD in determining the CVD risk of individuals with and without hypertension. If regression of CV damage is not achieved, the process advances to the development of $\mathrm{CV}$ events and progression of CKD to overt nephropathy and CVD. Although prevention strategies must be present along the continuum, interventions at this point should only retard the occurrence of $\mathrm{CV}$ and renal events [8]. This last stage represents the situation of further progression of vascular disease, leading to the appearance of symptomatic TOD (myocardial infarction, angina, stroke, transient ischemic attack, advanced chronic renal failure, and peripheral artery disease), which eventually will lead to end-stage renal disease (ESRD) or death. At this stage, the best we can do is to retard the likelihood of such events.

\section{Cardiovascular Disease Associated with Renal Disease}

Underlying the cardiorenal continuum is the pathophysiological continuum, which describes the progressive processes at molecular and cellular levels that manifest as clinical disease. A vast amount of research over the last two decades has provided considerably more knowledge regarding the therapeutic interventions that are able to intervene along the continuum.

Therefore, as CVD risk factors can be evaluated, the process begins. At this first stage of cardiorenal disease, preventative approaches are the most relevant strategies to disrupt disease progression [9]. In this sense, some data have demonstrated that high-risk patients without evidence of renal damage may benefit from early therapeutic intervention. The multicenter, double-blind, randomized Bergamo Nephrologic Diabetes Complications Trial (BENEDICT) assessed whether pharmacological intervention could prevent microalbuminuria in high-risk patients with no evidence of organ damage. The main results showed that intervention decreased the incidence of microalbuminuria [10]. Evidence from other ongoing trials will shed light on this issue, as will the Randomised Olmesartan and Diabetes Microalbuminuria Prevention (ROADMAP) studya placebo-controlled, multicenter, double-blind, parallel group study investigating the effect of the angiotensin receptor blocker $(\mathrm{ARB})$ olmesartan medoxomil on the incidence of microalbuminuria in hypertensive people with type 2 diabetes and an objective of blood pressure $<130 / 80 \mathrm{mmHg}$. In addition, ROADMAP will also analyze effects of olmesartan medoxomil on retinopathy and other microvascular circulations [11]. The results of the Diabetic Retinopathy Candesartan Trials (DIRECTs) are designed to examine primary (incidence) and secondary (progression) prevention of diabetic retinopathy when blocking angiotensin II type 1 receptors with the $\mathrm{ARB}$ candesartan in patients with normoalbuminuric, normotensive type 1 diabetes, and secondary prevention only in patients with normoalbuminuric, normotensive, or treated hypertensive type 2 diabetes. This trial series will also support prevention strategies to block advancement of the atherosclerotic process that leads to development of CV damage [12].

Optimal management in people with several risk factors is crucial, especially when hypertension is associated with other conditions. Awareness that several antihypertensive agents may exert undesirable metabolic effects has antihypertensive treatment trials to investigate the incidence of 
new-onset diabetes. Almost all such trials with new-onset diabetes as an endpoint have shown a significantly greater incidence in patients treated with diuretics and/or betablockers compared with angiotensin-converting enzyme inhibitors (ACEIs), ARBs, or calcium antagonists [13-16]. Angiotensin receptor antagonists [17] and ACEIs [13] have been shown to be associated with significantly fewer new diabetes cases than were calcium antagonists. The Ongoing Telmisartan Alone and in Combination with Ramipril Global Endpoint Trial (ONTARGET) is comparing telmisartan, ramipril, and their combination for preventing CVD morbidity and mortality in high-risk patients [18]. Telmisartan was the ARB selected for the ONTARGET because it provides sustained antihypertensive activity over the $24 \mathrm{~h}$ between doses [19]. The comparator, the ACEI ramipril, was selected because in the Heart Outcomes Prevention Evaluation (HOPE) trial, ramipril was proved to reduce the incidence of CV events in a similar patient population [20]. Patients enrolled in ONTARGET have vascular disease (coronary artery disease, peripheral arterial occlusive disease, stroke) or diabetes with TOD. The primary outcome is a composite endpoint of CVD, death, stroke, acute myocardial infarction, and hospitalization for congestive heart failure. A variety of renal endpoints have also been included. The Telmisartan Randomized Assessment Study in ACE-I-Intolerant Subjects with CV Disease (TRANSCEND) is a parallel study within the ONTARGET that is comparing the CV protective effect of telmisartan with placebo in patients intolerant of ACEIs [18]. The first results of this trial have been published and emphasize that the telmisartan was equivalent to ramipril in treating patients with vascular disease or high-risk diabetes and was better tolerated [21]. The combination of these two drugs was associated with more adverse events without an increased benefit. More evidence about prevention along the cardiorenal continuum is expected from this trial, including more than 150,000 patient-years of data. The Trial of Preventing Hypertension (TROPHY) hypothesized that early treatment with candesartan might prevent or delay hypertension onset. The main results showed that candesartan was better in preventing development of hypertension versus placebo [22]. The AngloScandinavian Cardiac Outcomes Trial (ASCOT) evaluated the benefits associated specifically with the use of statins among patients with hypertension [23]. Atorvastatin, which was added to the treatment therapy in more than 10,000 patients with hypertension and additional CVD risk factors and a serum total cholesterol $<6.5 \mathrm{mmol} / \mathrm{L}$, reduced serum total cholesterol by $19.9 \%$ compared with placebo. This was accompanied by substantial benefits both with regard to total CV and renal events (36\% reduction) and stroke (27\% reduction). The Avoiding Cardiovascular Events through Combination Therapy in Patients Living with Systolic Hypertension (ACCOMPLISH) trial was recently terminated prematurely because the predefined efficacy outcome was achieved and an interim analysis reported. The trial recruited more than 11,400 patients who received either amlodipine in combination with benazepril or hydrochlorothiazide in combination with benazepril. A primary composite endpoint of CVD morbidity or mortality was defined as death from
CV causes, fatal or nonfatal myocardial infarction or fatal or nonfatal stroke, revascularization, or unstable angina requiring hospitalization. Treatment with amlodipine/benazepril significantly reduced CVD morbidity and mortality compared with hydrochlorothiazide/benazepril (relative risk (RR) 0.80 ; 95\% confidence interval (CI) 0.71-0.90) [24]. Mechanical and chemical damages resulting from these interrelated CVD risk factors promote general progression of vascular damage that begins with endothelial dysfunction and atherosclerosis. This leads to end-organ damage, such as $\mathrm{LVH}$, subclinical atherosclerotic vascular damage, and kidney injury that can be detected by microalbuminuria and renal function derangement (estimated glomerular filtration rate (eGFR) $<60 \mathrm{~mL} / \mathrm{min} / 1.73 \mathrm{~m}^{2}$ or a slight increase in serum creatinine). At this second stage, vascular damage processes may be regressed, and inhibition of the rennin-angiotensin system (RAS) has been shown to be the most efficient pharmacological intervention along with strict control of CVD risk factors.

International guidelines devoted to arterial hypertension recognize microalbuminuria, elevated serum creatinine values, and reduced eGFR as major CVD risk factors that contribute to increased risk afforded by other coexisting factors [25-27]. The diagnosis of hypertension-induced renal damage in a hypertensive patient is usually based on reduced renal function and/or elevated urinary excretion of albumin. Renal function decline is classified in accordance with eGFR calculated by the abbreviated Modification of Diet in Renal Disease (MDRD) formula that assesses age, gender, race, and serum creatinine [28]. Values of eGFR $<60 \mathrm{~mL} / \mathrm{min} / 1.73 \mathrm{~m}^{2}$ indicate CKD stage 3, whereas values $<30$ and $15 \mathrm{~mL} / \mathrm{min} / 1.73 \mathrm{~m}^{2}$ indicate CKD stages 4 and 5, respectively [29]. The Cockcroft-Gault formula estimates creatinine clearance $(\mathrm{CrCl})$ and is based on age, gender, body weight, and serum creatinine [30]. This formula is applicable in the range $>60 \mathrm{~mL} / \mathrm{min}$, but it overestimates $\mathrm{CrCl}$ in $\mathrm{CKD}$ stages 3-5 [31]. Both procedures help to detect mildly impaired renal function in the face of serum creatinine values that are still in the normal range.

Reduction in GFR and increase in CVD risk may also be inferred from increased serum levels of cystatin C [32]. Whereas elevated serum creatinine concentration or low eGFR (or $\mathrm{CrCl}$ ) points to reduced rate of plasma filtered at the glomerular level, increased urinary albumin or protein excretion points to derangement in the glomerular filtration barrier, which allows increased albumin passage. Microalbuminuria has been shown to predict the development of overt diabetic nephropathy in those with either type 1 or type 2 diabetes [33]. However, only about $40 \%$ of those with type 2 diabetes will develop microalbuminuria, and, of those, approximately $50 \%$ will develop microalbuminuria in the following 10 years [34]. In contrast, in both diabetic and nondiabetic hypertensive patients, microalbuminuria, even below the threshold values currently considered [35], has been shown to predict CV events. Several studies report a continuous relationship between CVD—as well as non-CVD-mortality and urinary protein/creatinine ratios $>3.9 \mathrm{mg} / \mathrm{g}$ in men and $7.5 \mathrm{mg} / \mathrm{g}$ in women [36]. Thus, the term "microalbuminuria" may be misleading (because it 
falsely suggests a minor injury as well) and should, in theory, be replaced by the term "low-grade albuminuria" [37]. Microalbuminuria can be determined in spot urine samples ( $24 \mathrm{~h}$ or night-time urine samples are discouraged due to inaccuracy of urinary sampling) by indexing the urinary albumin concentration to the urinary creatinine concentration. Initial evidence concluding that microalbuminuria increases CVD risk came from observations involving highrisk patients [38]. Data from the HOPE study [39] confirmed the predictive value of microalbuminuria, which attained a predictive capacity similar to that of previous coronary artery disease and was equal for patients with and without accompanying diabetes. The relevance of urinary albumin excretion (UAE) as a CVD risk factor in patients with hypertension without diabetes and in the general population has also been demonstrated [40]. Some of these studies indicate that the relationship between urinary albumin and CVD risk is a continuum that starts below the established cutoff point indicated earlier. Definitely, both UAE and reduced GFR are independently associated with increased CVD risk, which is particularly elevated when both alterations coexist [41]. In fact, the prevalence of albuminuria, either micro or macro, increases as eGFR falls $<60 \mathrm{~mL} / \mathrm{min} / 1.73 \mathrm{~m}^{2}$ [42].

Patients developing ESRD are a minority in the group developing different forms of CKD. They could be considered survivors because CVD accounts for the majority of deaths of patients with CKD before the development of ESRD [43]. In turn, advanced CVD facilitates the development of CKD, and so the relationship between CKD and CVD becomes a vicious circle. That CKD and CVD are so closely related has resulted in increased interest in investigating the evolution of renal function in trials involving patients with hypertension, as well as those with heart failure and postmyocardial infarction. This interest is fully justified, as, in all these situations, renal function alterations are predictive for the development of CV events or death.

Even from the early stages, CKD adds to CVD risk in any patient with hypertension and in any patient presenting with established forms of CVD [44]. Reduction of CV events in the CKD population requires the implementation of effective integral therapeutic interventions that protect both the kidney and the CV system. These interventions have to be implemented in the very initial stages of CKD, and strict blood pressure control is imperative in any patient with an elevated global CVD risk and high blood pressure. In the absence of other CVD risk factors, elevated blood pressure levels are required in order to consider patients as having high-added CVD risks. In contrast, only high-normal blood pressure levels or even lower values are required for the same evaluation when patients present with three or more associated CVD risk factors, TOD, diabetes, or associated clinical conditions. Accordingly, patients with hypertension and a high-added level of CVD risk can be found in any of the three stages of the CV and renal disease continuum. As soon as renal function exhibits minor derangements, CVD risk continues to increase until ESRD develops.

As renal function declines, TOD appears and CKD adds several clinical characteristics that raise the possibility of a
$\mathrm{CV}$ event as atherosclerotic disease progresses. CKD-induced anemia and secondary hyperparathyroidism globally worsen outcomes in patients with and without myocardiopathies, and correction of these conditions is crucial to reduce absolute CVD risk [45, 46]. Among patients who referred to the authors' hypertension unit, $7.6 \%$ had a decreased renal function according to serum creatinine levels, and 25\% had a decreased $\mathrm{CrCl}$ [47]. Community-based longitudinal studies demonstrated that CKD is an independent risk factor for the composite study outcome, including myocardial infarction, fatal congestive heart failure, stroke, and death [48]. In patients with essential hypertension and normal renal function (defined as eGFR $>90 \mathrm{~mL} / \mathrm{min} / 1.73 \mathrm{~m}^{2}$ ), those who developed CKD during 13 years of followup had a CV event rate 2.5 times higher than did those with preserved renal function [49]. As widely evidenced in the hypertensive population, the higher the CVD risk, the higher the CKD prevalence [50].

Evidence for the relationship between renal dysfunction and adverse CV events was initially documented in the ESRD population in whom the incidence of CVD death is elevated. Around 50\% of individuals with ESRD die from a CVD-a CVD mortality rate much higher than the age-adjusted CVD mortality rate in the general population. This discrepancy is present across all ages, but it is most marked in the younger age group, in which the CVD mortality rate is $>300$-fold in ESRD patients compared with age-matched controls with normal renal function [51]. By the time ESRD occurs, 40\% of patients have evidence of $\mathrm{CHF}$, and $85 \%$ of those patients have abnormal LV structure and function

The relationship between renal disease and CVD mortality has also been shown to extend to patients with more moderate degrees of renal impairment. Indeed, the majority of patients with eGFR $<60 \mathrm{~mL} / \mathrm{min} / 1.73 \mathrm{~m}^{2}$ die from CVDrelated causes rather than progressing to ESRD. In addition, evidence of structural and functional cardiac abnormalities has been demonstrated. Data about cardiac structure in the renal insufficiency population has been described with echocardiographic techniques and comparable criteria for diagnosing LVH, detecting an LVH prevalence of $16 \%$ in patients with $\mathrm{CrCl}$ of $>30 \mathrm{~mL} / \mathrm{min}$ and $38 \%$ in those with $\mathrm{CrCl}<30 \mathrm{~mL} / \mathrm{min}$ [52]. Therefore, $\mathrm{LVH}$ is common in patients with renal insufficiency even before they progress to dialysis, and so prevalence of LVH correlates with the degree of renal functional deterioration. Many reports have shown that the relationship between renal impairment and increased CVD mortality rate extends across the spectrum of renal dysfunction to cover the mildest degree of renal disease. Furthermore, this relationship appears to be maintained through populations with broadly diverse degrees of baseline $\mathrm{CV}$ health. LVH is an independent predictor of unfavorable prognosis in the hypertensive population, and, in the LIFE study, its relationship with albumin excretion was reported as being independent of age, blood pressure, diabetes mellitus, race, serum creatinine level, or smoking [53]. The prevalence of microalbuminuria was approximately twofold higher in patients with hypertension and eccentric or concentric LVH and minimally elevated in the group with concentric LV remodelling compared with patients with normal LV 
geometry. Although the clinical significance of impaired renal function and LVH in patients with hypertension is not yet fully understood, numerous reports link renal albumin leakage with morbidity and mortality.

The LIFE study also showed that the simple measurement of UACR further refines risk stratification by LV geometry and that patients with LVH have an increased risk of also having albuminuria, a situation that should be further investigated to improve treatment and counselling. The risk for CVD endpoints increases in a stepwise trend with higher values for UACR in patients with diabetes. Data indicate that albuminuria at a lower level than that usually used as a cut point in patients with diabetes defines patients at increased risk of CVD morbidity and mortality. UACR did not predict the risk of myocardial infarction. Perhaps diabetes itself is a strong predictor for CVD morbidity and mortality, partly overlapping the influence of albuminuria as a risk factor in the population with rather low levels of albuminuria. Other studies suggest that albuminuria at levels below established values is a risk factor for CHF in patients with and without diabetes, signifying that the relationship between albuminuria and CVD risk from other populations cannot be directly applied to nondiabetic hypertensive patients [54].

Global (all risk factors) (AU: global, meaning worldwide, or treating all risk factors in the individual patient?) and strict control of the sum of CVD risk factors and therapeutic action in order to regress already established vascular damage must be the cornerstone of the medical strategy, because, if not stopped, the cardiorenal continuum progresses to CKD (proteinuria, eGFR $<30 \mathrm{~mL} / \mathrm{min} / 1.73 \mathrm{~m}^{2}$ ), overt CVD, and stroke. Interventions at this point are focused on delayed development of CV and renal events [27]. CV events and consequent death are dramatically reduced when UACR is decreased and GFR decline is avoided. If renal decline progresses to the final stage, proteinuria will occur. In type 2 diabetes, data from the Reduction of Endpoints in NIDDM with the Angiotensin II Antagonist Losartan (RENAAL) trial showed that changes in albuminuria in the first 6 months of therapy were approximately linearly related to the degree of long-term renal protection: every $50 \%$ reduction in albuminuria in the first 6 months was associated with a $45 \%$ reduction in the risk for ESRD during later followup [55]. Furthermore, a secondary analysis of the Irbesartan in Diabetic Nephropathy Trial (IDNT) demonstrated that the risk for renal failure was reduced during the first year of the study when there were increases in proteinuria [56]. Subsequently, these two studies (IDNT and RENAAL) demonstrated that an ARB (irbesartan or losartan) was more effective than conventional therapy or a calcium channel blocker in slowing progression of nephropathy, regardless of blood pressure control. Moreover, secondary analyses of these two large trials demonstrated that there was some interaction between the effect of the ARB and the levels of blood pressure that were achieved. It can also be concluded that optimal levels of blood pressure tended to magnify the renoprotective effects of ARB in both trials. In the large cohort of patients with hypertension, microalbuminuria, and type 2 diabetes who participated in the Microalbuminuria, Cardiovascular, and Renal Outcomes-Heart Outcomes Prevention Evaluation
(MICRO-HOPE), the ACEI compared with other treatments was more effective in reducing the incidence of overt nephropathy [57]. Furthermore, the Irbesartan in Patients with Type 2 Diabetes and Microalbuminuria (IRMA-2) study showed that treatment with the ARB irbesartan was much more effective than conventional therapy at both preventing the development of clinical proteinuria and favoring regression to normoalbuminuria in patients with microalbuminuria and type 2 diabetes, despite similar blood pressure control [58].

\section{Global Therapeutic Approach Focused on Renal Outcomes}

CKD progression, that is, reduced GFR, occurs at a variable rate, with a faster rate of decline generally noted among patients with diabetic nephropathy due to the presence of proteinuria. Several therapeutic options have been shown to be efficient in slowing the rate of renal function decline. Among these therapeutic treatments are blood-pressurereducing drugs-preferably ACEIs and/or angiotensin II antagonists-low-salt and low-protein diets, and lipidlowering drugs [59]. Unfortunately, for such treatments to be most efficacious and in agreement with the European Society of Hypertension/European Society of Cardiology guidelines, it is necessary to identify patients in an early stage of disease before significant loss of renal function has occurred. Such identification is simplified by estimating GFR and measuring microalbuminuria in any patient with hypertension. UACR levels of approximately $>2 \mathrm{mg} / \mathrm{g}$ or an estimated excretion rate of $2 \mathrm{mg} / \mathrm{day}$ are significantly associated with death from CVD, myocardial infarction, stroke, and elevated blood pressure. As a result, reductions in albuminuria levels during treatment translate to regression of a number of vascular abnormalities in hypertension and thus a decrease in risk in general. In patients with type 2 diabetes and diabetic nephropathy, and also in patients with nondiabetic renal disease, data indicate that the extent of decreases in albuminuria during renin-angiotensinaldosterone system intervention is associated with the degree of renal protection and also the degree of reduced CVD risk [60]. Reductions in both systolic and diastolic blood pressure are important in reducing albuminuria levels. Despite the firm relationship between blood pressure values and albuminuria, ACEIs and ARBs exhibit a more marked capacity to reduce microalbuminuria in patients with hypertension compared with a number of different therapeutic interventions, such as calcium antagonists, beta-blockers, or diuretics [61].

\section{References}

[1] J. E. Hall, M. W. Brands, and E. W. Shek, "Central role of the kidney and abnormal fluid volume control in hypertension," Journal of Human Hypertension, vol. 10, no. 10, pp. 633-639, 1996.

[2] R. W. Schrier and W. T. Abraham, "Hormones and hemodynamics in heart failure," New England Journal of Medicine, vol. 341, no. 8, pp. 577-585, 1999. 
[3] J. R. Brown, R. P. Cochran, B. J. Leavitt et al., "Multivariable prediction of renal insufficiency developing after cardiac surgery," Circulation, vol. 116, no. 11, pp. I139-I143, 2007.

[4] R. N. Foley, A. M. Murray, S. Li et al., "Chronic kidney disease and the risk for cardiovascular disease, renal replacement, and death in the United States medicare population, 1998 to 1999," Journal of the American Society of Nephrology, vol. 16, no. 2, pp. 489-495, 2005.

[5] L. Gruberg, N. J. Weissman, R. Waksman et al., "Comparison of outcomes after percutaneous coronary revascularization with stents in patients with and without mild chronic renal insufficiency," American Journal of Cardiology, vol. 89, no. 1, pp. 54-57, 2002.

[6] V. J. Dzau, E. M. Antman, H. R. Black et al., "The cardiovascular disease continuum validated: clinical evidence of improved patient outcomes: part II: clinical trial evidence (acute coronary syndromes through renal disease) and future directions," Circulation, vol. 114, no. 25, pp. 2871-2891, 2006.

[7] C. Ronco, P. McCullough, S. D. Anker et al., "Cardio-renal syndromes: report from the consensus conference of the acute dialysis quality initiative," European Heart Journal, vol. 31, no. 6, pp. 703-711, 2010.

[8] L. M. Ruilope, S. E. Kjeldsen, A. de la Sierra et al., "The kidney and cardiovascular risk-implications for management: a consensus statement from the European Society of Hypertension," Blood Pressure, vol. 16, no. 2, pp. 72-79, 2007.

[9] W. F. Keane, "Metabolic pathogenesis of cardiorenal disease," American Journal of Kidney Diseases, vol. 38, no. 6, pp. 13721375, 2001.

[10] P. Ruggenenti, A. Fassi, A. P. Ilieva et al., "Preventing microalbuminuria in type 2 diabetes," New England Journal of Medicine, vol. 351, no. 19, pp. 1941-1951, 2004.

[11] H. Haller, G. C. Viberti, A. Mimran et al., "Preventing microalbuminuria in patients with diabetes: rationale and design of the Randomised Olmesartan and Diabetes Microalbuminuria Prevention (ROADMAP) study," Journal of Hypertension, vol. 24, no. 2, pp. 403-408, 2006.

[12] A. K. Sjølie, R. Bilous, N. Chaturvedi et al., "The DIabetic REtinopathy Candesartan Trials (DIRECT) Programme: baseline characteristics," Journal of the Renin-AngiotensinAldosterone System, vol. 6, no. 1, pp. 25-32, 2005.

[13] S. Julius, S. E. Kjeldsen, M. Weber et al., "Outcomes in hypertensive patients at high cardiovascular risk treated with regimens based on valsartan or amlodipine: the VALUE randomised trial," Lancet, vol. 363, no. 9426, pp. 2022-2031, 2004.

[14] H. Lithell, L. Hansson, I. Skoog et al., "The Study on Cognition and Prognosis in the Elderly (SCOPE). Principal results of a randomised double-blind intervention trial," Journal of Hypertension, vol. 21, pp. 875-886, 2003.

[15] L. Hansson, L. H. Lindholm, L. Niskanen et al., "Effect of angiotensin-converting-enzyme inhibition compared with conventional therapy on cardiovascular morbidity and mortality in hypertension: the Captopril Prevention Project (CAPPP) randomised trial," Lancet, vol. 353, no. 9153, pp. 611-616, 1999.

[16] L. Hansson, T. Hedner, P. Lund-Johansen et al., "Randomised trial of effects of calcium antagonists compared with diuretics and $\beta$-blockers on cardiovascular morbidity and mortality in hypertension: the Nordic Diltiazem (NORDIL) study," Lancet, vol. 356, no. 9227, pp. 359-365, 2000.
[17] The ALLHAT officers and coordinators for the ALLHAT Collaborative Research Group, "Major outcomes in high-risk hypertensive patients randomized to angiotensin-converting enzyme inhibitor or calcium channel blocker vs diuretic: the Antihypertensive and Lipid-Lowering treatment to prevent Heart Attack Trial (ALLHAT)," Journal of the American Medical Association, vol. 288, pp. 2981-2997, 2002.

[18] The ONTARGET/TRANSCEND investigators, "Rationale, design, and baseline characteristics of 2 large, simple, randomized trials evaluating telmisartan, ramipril, and their combination in high-risk patients: the Ongoing Telmisartan Alone and in Combination with Ramipril Global Endpoint Trial/Telmisartan Randomized Assessment Study in ACE-IIntolerant Subjects with Cardiovascular Disease (ONTARGET/TRANSCEND) trials," American Heart Journal, vol. 148, pp. 52-61, 2004.

[19] A. J. Battershill and L. J. Scott, "Telmisartan: a review of its use in the management of hypertension," Drugs, vol. 66, no. 1, pp. 51-83, 2006.

[20] S. Yusuf, P. Sleight, J. Pogue et al., "Effects of an angiotensinconverting-enzyme inhibitor, ramipril, on cardiovascular events in high-risk patients. The Heart Outcomes Prevention Evaluation Study Investigators," New England Journal of Medicine, vol. 342, pp. 145-153, 2000.

[21] S. Yusuf, K. K. Teo, J. Pogue et al., "Telmisartan, ramipril, or both in patients at high risk for vascular events," New England Journal of Medicine, vol. 358, no. 15, pp. 1547-1559, 2008.

[22] S. Julius, S. D. Nesbitt, B. M. Egan et al., "Feasibility of treating prehypertension with an angiotensin-receptor blocker," New England Journal of Medicine, vol. 354, no. 16, pp. 1685-1697, 2006.

[23] P. S. Sever, B. Dahlof, N. R. Poulter et al., "ASCOT investigators. The prevention of coronary events and stroke with atorvastatin in hypertensive subjects with average or below average cholesterol levels. The Anglo-Scandinavian Cardiac Outcomes Trial: Lipid Lowering Arm (ASCOT:LLA)," Lancet, vol. 361, pp. 1149-1158, 2003.

[24] K. Jamerson, M. A. Weber, G. L. Bakris et al., "Benazepril plus amlodipine or hydrochlorothiazide for hypertension in highrisk patients," New England Journal of Medicine, vol. 359, no. 23, pp. 2417-2428, 2008.

[25] A. V. Chobanian, G. L. Bakris, H. R. Black et al., "The seventh report of the joint national committee on prevention, detection, evaluation, and treatment of high blood pressure: the JNC 7 report," Journal of the American Medical Association, vol. 289, no. 19, pp. 2560-2572, 2003.

[26] G. Mancia, G. De Backer, A. Dominiczak et al., "2007 Guidelines for the Management of Arterial Hypertension: The Task Force for the Management of Arterial Hypertension of the European Society of Hypertension (ESH) and of the European Society of Cardiology (ESC)," Journal of Hypertension, vol. 25, no. 6, pp. 1105-1187, 2007.

[27] M. Tonelli, N. Wiebe, B. Culleton et al., "Chronic kidney disease and mortality risk: a systematic review," Journal of the American Society of Nephrology, vol. 17, no. 7, pp. 2034-2047, 2006.

[28] S. Hallan, A. Asberg, M. Lindberg, and H. Johnsen, "Validation of the modification of diet in renal disease formula for estimating GFR with special emphasis on calibration of the serum creatinine assay," American Journal of Kidney Diseases, vol. 44, no. 1, pp. 84-93, 2004.

[29] S. Moe, T. Drueke, J. Cunningham et al., "Kidney Disease: Improving Global Outcomes (KDIGO). Definition and classification of chronic kidney disease: a position statement 
from Kidney Disease: Improving Global Outcomes (KDIGO)," Kidney International, vol. 67, pp. 2089-2100, 2005.

[30] D. W. Cockcroft and M. H. Gault, "Prediction of creatinine clearance from serum creatinine," Nephron, vol. 16, no. 1, pp. 31-41, 1976.

[31] L. M. Ruilope, A. Zanchetti, S. Julius et al., "Prediction of cardiovascular outcome by estimated glomerular filtration rate and estimated creatinine clearance in the high-risk hypertension population of the VALUE trial," Journal of Hypertension, vol. 25, no. 7, pp. 1473-1479, 2007.

[32] M. G. Shlipak, R. Katz, M. J. Sarnak et al., "Cystatin C and prognosis for cardiovascular and kidney outcomes in elderly persons without chronic kidney disease," Annals of Internal Medicine, vol. 145, no. 4, pp. 237-246, 2006.

[33] H. H. Parving, "Initiation and progression of diabetic nephropathy," New England Journal of Medicine, vol. 335, no. 22, pp. 1682-1683, 1996.

[34] M. A. Gall, P. Hougaard, K. Borch-Johnsen, and H. H. Parving, "Risk factors for development of incipient and overt diabetic nephropathy in patients with non-insulin dependent diabetes mellitus: prospective, observational study," British Medical Journal, vol. 314, no. 7083, pp. 783-788, 1997.

[35] J. Redon and B. Williams, "Microalbuminuria in essential hypertension: redefining the threshold," Journal of Hypertension, vol. 20, no. 3, pp. 353-355, 2002.

[36] H. L. Hillege, V. Fidler, G. F. H. Diercks et al., "Urinary albumin excretion predicts cardiovascular and noncardiovascular mortality in general population," Circulation, vol. 106, no. 14, pp. 1777-1782, 2002.

[37] J. Ärnlöv, J. C. Evans, J. B. Meigs et al., "Low-grade albuminuria and incidence of cardiovascular disease events in nonhypertensive and nondiabetic individuals: the framingham heart study," Circulation, vol. 112, no. 7, pp. 969-975, 2005.

[38] E. M. Damsgaard, A. Froland, O. D. Jorgensen, and C. E. Mogensen, "Microalbuminuria as predictor of increased mortality in elderly people," British Medical Journal, vol. 300, no. 6720 , pp. 297-300, 1990.

[39] H. C. Gerstein, J. F. E. Mann, Q. Yi et al., "Albuminuria and risk of cardiovascular events, death, and heart failure in diabetic and nondiabetic individuals," Journal of the American Medical Association, vol. 286, no. 4, pp. 421-426, 2001.

[40] S. Agewall, J. Wikstrand, S. Ljungman, and B. Fagerberg, "Usefulness of microalbuminuria in predicting cardiovascular mortality in treated hypertensive men with and without diabetes mellitus," American Journal of Cardiology, vol. 80, no. 2, pp. 164-169, 1997.

[41] P. A. Sarafidis and G. L. Bakris, "Microalbuminuria and chronic kidney disease as risk factors for cardiovascular disease," Nephrology Dialysis Transplantation, vol. 21, no. 9, pp. 2366-2374, 2006.

[42] J. Segura and L. M. Ruilope, "Minor abnormalities of renal function: a situation requiring integrated management of cardiovascular risk," Fundamental and Clinical Pharmacology, vol. 19, no. 4, pp. 429-437, 2005.

[43] E. F. Elsayed, H. Tighiouart, J. Griffith et al., "Cardiovascular disease and subsequent kidney disease," Archives of Internal Medicine, vol. 167, no. 11, pp. 1130-1136, 2007.

[44] L. M. Ruilope, V. Lahera, J. L. Rodicio, and J. C. Romero, "Are renal hemodynamics a key factor in the development and maintenance of arterial hypertension in humans?" Hypertension, vol. 23, no. 1, pp. 3-9, 1994.

[45] P. T. Vlagopoulos, H. Tighiouart, D. E. Weiner et al., "Anemia as a risk factor for cardiovascular disease and all-cause mortality in diabetes: the impact of chronic kidney disease,"
Journal of the American Society of Nephrology, vol. 16, no. 11, pp. 3403-3410, 2005.

[46] S. M. Moe, "Vascular calcification and renal osteodystrophy relationship in chronic kidney disease," European Journal of Clinical Investigation, vol. 36, no. 2, pp. 51-62, 2006.

[47] J. Segura, C. Campo, and L. M. Ruilope, "How relevant and frequent is the presence of mild renal insufficiency in essential hypertension?" Journal of Clinical Hypertension, vol. 4, no. 5, pp. 332-336, 2002.

[48] D. E. Weiner, H. Tighiouart, M. G. Amin et al., "Chronic kidney disease as a risk factor for cardiovascular disease and all-cause mortality: a pooled analysis of community-based studies," Journal of the American Society of Nephrology, vol. 15, no. 5, pp. 1307-1315, 2004.

[49] J. Segura, C. Campo, P. Gil et al., "Development of chronic kidney disease and cardiovascular prognosis in essential hypertensive patients," Journal of the American Society of Nephrology, vol. 15, no. 6, pp. 1616-1622, 2004.

[50] L. M. Ruilope, "The kidney as a sensor of cardiovascular risk in essential hypertension," Journal of the American Society of Nephrology, vol. 13, no. 3, pp. S165-S168, 2002.

[51] E. L. Schiffrin, M. L. Lipman, and J. F. E. Mann, "Chronic kidney disease: effects on the cardiovascular system," Circulation, vol. 116, no. 1, pp. 85-97, 2007.

[52] B. Tucker, F. Fabbian, M. Giles, R. C. Thuraisingham, A. E. G. Raine, and L. R. I. Baker, "Left ventricular hypertrophy and ambulatory blood pressure monitoring in chronic renal failure," Nephrology Dialysis Transplantation, vol. 12, no. 4, pp. 724-728, 1997.

[53] K. Wachtell, M. H. Olsen, B. Dahlöf et al., "Microalbuminuria in hypertensive patients with electrocardiographic left ventricular hypertrophy: the LIFE study," Journal of Hypertension, vol. 20, no. 3, pp. 405-412, 2002.

[54] K. Klausen, K. Borch-Johnsen, B. Feldt-Rasmussen et al., "Very low levels of microalbuminuria are associated with increased risk of coronary heart disease and death independently of renal function, hypertension, and diabetes," Circulation, vol. 110, no. 1, pp. 32-35, 2004.

[55] B. M. Brenner, M. E. Cooper, D. de Zeeuw et al., "Effects of losartan on renal and cardiovascular outcomes in patients with type 2 diabetes and nephropathy," New England Journal of Medicine, vol. 345, no. 12, pp. 861-869, 2001.

[56] E. J. Lewis, L. G. Hunsicker, W. R. Clarke et al., "Renoprotective effect of the angiotensin-receptor antagonist irbesartan in patients with nephropathy due to type 2 diabetes," New England Journal of Medicine, vol. 345, no. 12, pp. 851-860, 2001.

[57] Heart Outcomes Prevention Evaluation Study investigators, "Effects of ramipril on cardiovascular and microvascular outcomes in people with diabetes mellitus: results of the HOPE study and MICRO-HOPE substudy," Lancet, vol. 355, pp. 253-259, 2000.

[58] H. H. Parving, H. Lehnert, J. Brochner-Mortensen, R. Gomis, S. Andersen, and P. Arner, "The effect of irbesartan on the development of diabetic nephropathy in patients with type 2 diabetes," New England Journal of Medicine, vol. 345, no. 12, pp. 870-878, 2001.

[59] H. H. H. Feringa, S. E. Karagiannis, M. Chonchol et al., "Lower progression rate of end-stage renal disease in patients with peripheral arterial disease using statins or angiotensinconverting enzyme inhibitors," Journal of the American Society of Nephrology, vol. 18, no. 6, pp. 1872-1879, 2007. 
[60] T. H. Jafar, P. C. Stark, C. H. Schmid et al., "Progression of chronic kidney disease: the role of blood pressure control, proteinuria, and angiotensin-converting enzyme inhibition. A patient-level meta-analysis," Annals of Internal Medicine, vol. 139, no. 4, pp. 244-I36, 2003.

[61] L. M. Ruilope, J. M. Alcazar, E. Hernandez, M. Praga, V. Lahera, and J. L. Rodicio, "Long-term influences of antihypertensive therapy on microalbuminuria in essential hypertension," Kidney International, Supplement, no. 45, pp. S171-S173, 1994. 


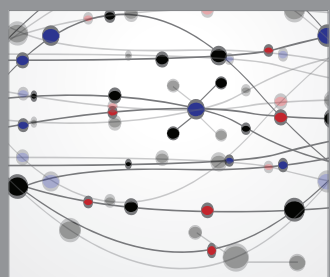

The Scientific World Journal
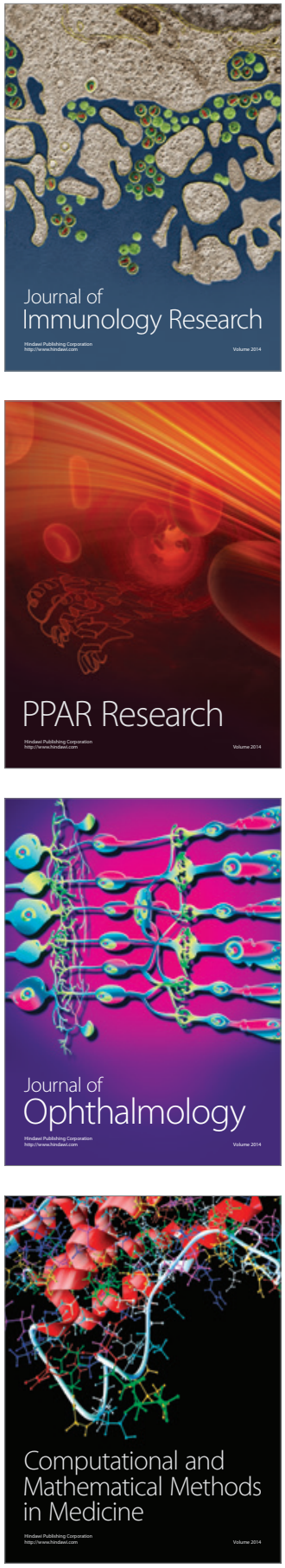

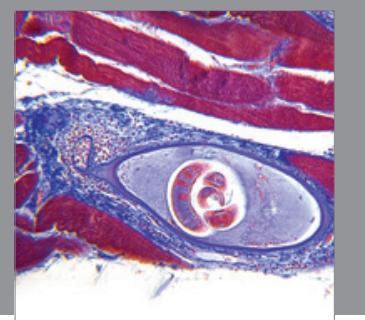

Gastroenterology

Research and Practice
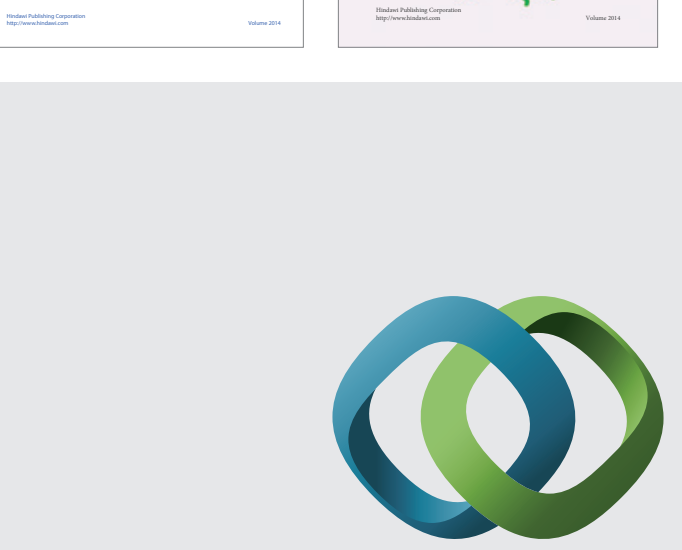

\section{Hindawi}

Submit your manuscripts at

http://www.hindawi.com
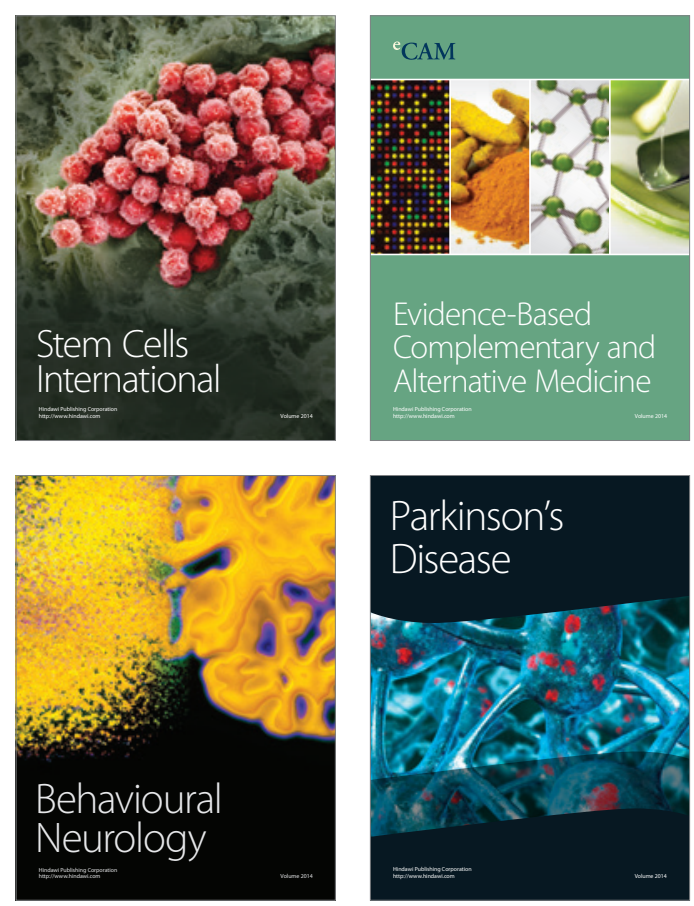

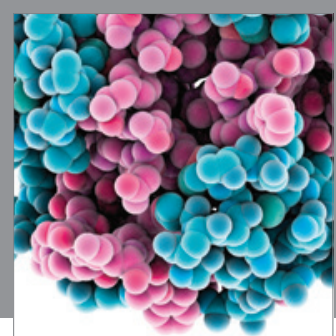

Journal of
Diabetes Research

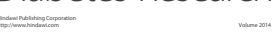

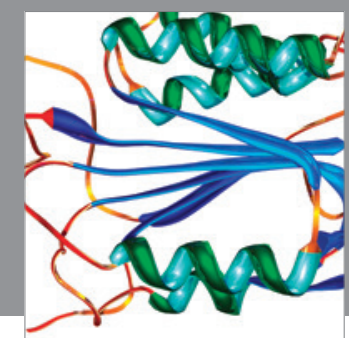

Disease Markers
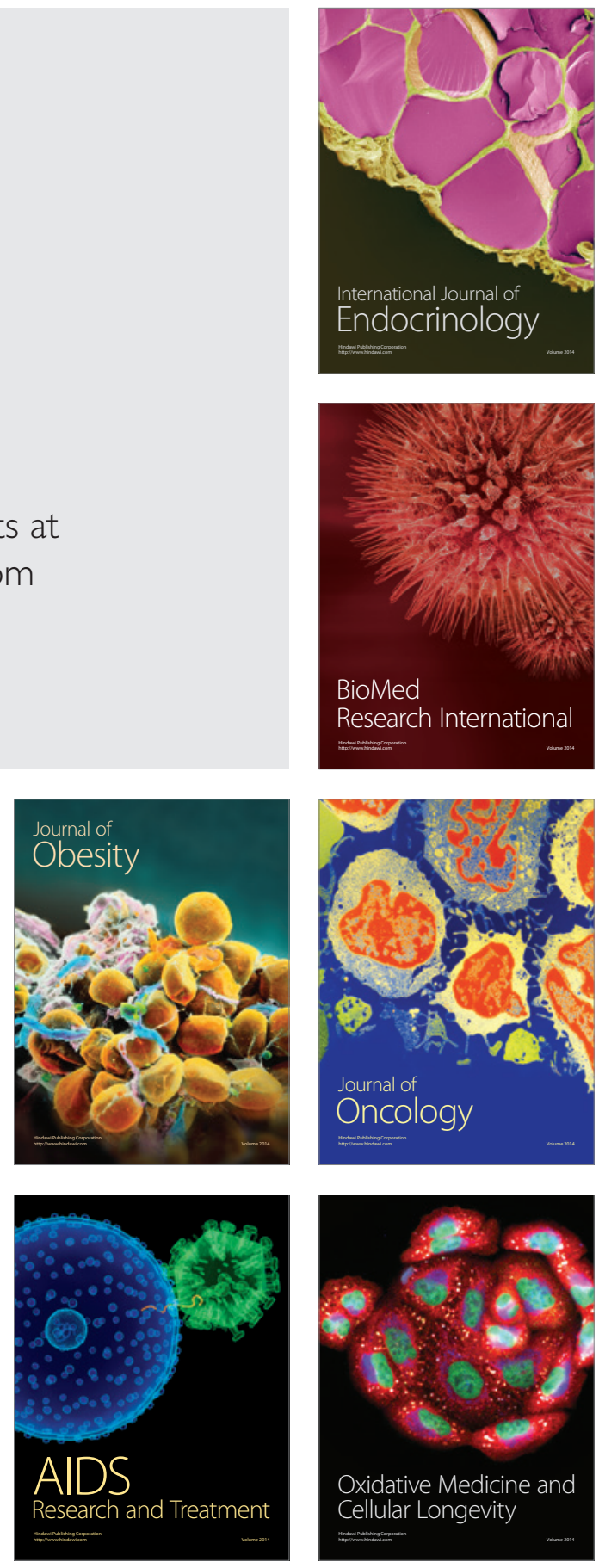